\title{
Exome Sequencing Identifies a Novel GUCY2D Mutation in an Iranian Family with Leber Congenital Amaurosis-1: A Case Report
}

Mostafa Neissi ( $\square$ iammostafaneissi@gmail.com )

Islamic Azad University https://orcid.org/0000-0002-9359-2054

Adnan Issa Al-Badran

University of Basrah

Javad Mohammadi-AsI

Ahvaz Jondishapour University of Medical Sciences

\section{Research Article}

Keywords: Leber congenital amaurosis, GUCY2D, Novel mutation, whole exome sequencing (WES), Case report

Posted Date: November 8th, 2021

DOI: https://doi.org/10.21203/rs.3.rs-586904/v1

License: (c) (i) This work is licensed under a Creative Commons Attribution 4.0 International License. Read Full License

Version of Record: A version of this preprint was published at Egyptian Journal of Medical Human Genetics on January 22nd, 2022. See the published version at https://doi.org/10.1186/s43042-02200217-9. 


\section{Abstract}

Background: Leber congenital amaurosis (LCA), the severe form of inherited retinal degenerative disorder is a prevalent disorder in the first year of life. Recently, genetic studies discovered that different gene mutations are responsible for LCA clinical manifestations.

Case presentation: In this study, we applied whole exome sequencing (WES) to identify probable gene defects in an Iranian girl with LCA-1. We found a novel disease-causing GUCY2D gene mutation (c.2348T>C; p.L783P), located in exon 12 (NM_000180), causing a missense mutation that has been changed the coding protein. The WES-identified variant was confirmed by Sanger sequencing for the patient and her healthy parents. Submitted to genetic counseling that the patient was 1 -year old and blindness from birth.

Conclusions: Our findings establish that this detected GUCY2D-p.L783P mutation is the pathogenic variant for LCA-1. This is the first genetic study indicating that c.2348T $>C$ missense mutation in the homozygous state in GUCY2D gene is responsible for the LCA-1 phenotype.

\section{Background}

Leber congenital amaurosis (LCA) is the most severe form of inherited retinal dystrophy that diagnosed with blindness or severe visual impairment before the first year of age $(1,2)$. LCA is an autosomal recessive disorder with the prevalence rate of 1 patient in every 80,000 people around the world (3).

Etiologically, childhood blindness can be triggered from different risk factors such as genetic and chromosomal abnormalities, intrauterine infections, etc (4). Studies have shown that genetic factors are responsible for more than $50 \%$ of all types of eye diseases (5). Genetic eye diseases include a large number of ocular complications that can be passed from parents to children (6)

Previously it has been identified that genetic abnormality in a group of genes included GUCY2D, RPE65, RPGRIP1, AIPL 1, CRB1, NMNAT1, and CEP290 associated with LCA cases, however more studies demonstrated that there are 26 genes related to the $\operatorname{LCA}(7,8)$. On the other hand, it has been determined that recessive and dominant mutations in the $G U C Y 2 D$ gene, were the underlying defects in LCA- 1 and cone-rod degeneration, respectively $(9,10)$. The GUCY2D gene is composed of 20 exons, located on the short arm of chromosome 17 (17p13.1) and encodes a 1,103-amino acids protein named retinal guanylyl cyclase-1 (RetGC-1) (10).

Moreover, the whole exome sequencing (WES) technique is a perfect diagnostic method in these cases that compares the obtained results with the reference sequences. Therefore, it is able to identify diseasecausing mutations in affected patients (11).

We reported here an Iranian patient affected by LCA-1 with blindness. To identify the underlying genetic defect, in this case, we performed WES followed by a targeted sequencing approach. 


\section{Case Presentation}

We have enrolled an Iranian family with LCA-1 from the Ahvaz (Khuzestan province, Iran). The patient was a 1-year-old girl with no light perception (NLP) blindness (Fig. 1A). Clinical examination was performed by Neuro-ophthalmologists and confirmed as a blindness case by visual evoked potentials (VEPs) test. The patient's problem was blindness at the beginning of her life, as her parents were mentioned to the genetic counseling. Other clinical complication was retinal degeneration throughout the retina in both eyes. She was born to consanguineous normal parents and there was no family history of inherited diseases such as LCA.

Total genomic DNA (gDNA) was extracted from peripheral blood leukocytes sample of the patient and her family members using a standard salting-out protocol. WES (Macrogen, Seoul, South Korea) was conducted to detect mutations in particular genes to hereditary this type of eye disease in the gDNA of the proband. A novel single-nucleotide variant (NM_000180: c.2348T>C; p.L783P) was detected in exon 12 of the GUCY2D gene. No mutation was detected in other genes.

The frequency (below 1\%) of the detected variants were checked in the 1000 genomes database (https://www.internationalgenome.org/), Exome Aggregation Consortium (ExAC), and Single Nucleotide Polymorphism Database (dbSNP). In silico analyses using Sort Intolerated From Tolerated (SIFT), Polymorphism Phenotyping v2 (PolyPhen-2), and Mutation Taster predicted that L783P variant would be damaging and disease-causing. Also, the mutation has been classified as likely pathogenic based on American College of Medical Genetics and Genomics (ACMG) guidelines. Finally, the candidate variant was checked by both ClinVar (https://www.ncbi.nlm.nih.gov/clinvar/) and the Human Gene Mutation Database (HGMD, http://www.hgmd.cf.ac.uk/ac/index.php). This is the first report of mutation of the GUCY2D gene in a patient affected by LCA-1.

The candidate mutation (c.2348T >C) was confirmed by sanger sequencing for the patient and family members. In order to amplify the GUCY2D-Specific genomic DNA fragments, the polymerase chain reaction (PCR) method was used. The results demonstrated that the affected case was homozygous for this novel mutation and her parents carried a heterozygous GUCY2D c.2348T>C (p.L783P) variant (Fig. 1B). This missense mutation causes variation in amino acid from leucine to proline (CTG>CCG) at codon 783 (Fig. 1C).

\section{Discussion}

LCA is a major concern among different types of inherited retinal blindness due to its devastating severity (1). Although genetic consulting for consanguine parents is highly recommended, we are still witnessing the birth of babies with congenital disorders $(1,3,12)$. So far, studies on genetic defects of LCA indicated that about $70 \%$ of patients suffered from a molecular disease-causing event. As the recent efforts focused on the new therapeutic strategies such as gene replacement therapy, finding the underlying genetic problem in affected patients is a very important issue (1). 
Hence, in this study, we investigated for the disease-causing mutation in a 1-year-old affected girl, who was referred to medical genetics for no light perception (NLP) blindness. The patient was belonging to consanguineous healthy parents with no history of inherited disorders. The WES was applied to identify the impaired gene in this case and a homozygous missense mutation (c.2348T >C; p.Leu783Pro) in the exon 12 of the GUCY2D gene was detected, followed by heterozygous mutations in her consanguine parents that resulted in homogeneity in their child.

The GUCY2D gene located in chromosome 17, encodes retGC-1 protein that plays a critical role in restoring photoreceptor sensitivity by converting guanosine triphosphate (GTP) to cyclic guanosine monophosphate (cGMP) (10). It was declared that GUCY2D gene mutations accounted for about $6-21 \%$ of all LCA cases (13). Considering the most of 127-point mutations detected so far in GUCY2D gene can affect all retGC-1 domains and subsequently result in LCA phenotype $(9,13)$, our finding also added another a novel missense mutation in GUCY2D gene leading to LCA-1 disorder.

We proposed that this novel missense mutation encodes an impaired form of retGC-1 protein that probably defects in function or stability resulting in LCA-1 clinical manifestations. In addition, it has been described previously that the LCA-1 phenotype caused by mutations in the retGC-1, dramatically reduced retGC-1 activity $(9,13)$. So that, in a study, Xue Feng et al. evaluated a Chinese family with LCA-1 to determine underlying genetic defect and showed that there were 3 novel mutation in the GUCY2D gene result in truncated protein or activity reduction (13). In another investigation, Libe Gradstein et al. described a novel mutation in GUCY2D gene as well as other known mutations in eight affected LCA patients. In addition, molecular modeling approaches implied that loss of retGC-1 helical structure because of the c.2129C>T; p.Ala710Val mutation in the GUCY2D gene, which is likely to affect the catalytic center (14).

Finally, the GUCY2D-p.L783P mutation was identified in an Iranian patient affected by LCA-1 and hasn't been previously reported in any of the mutation databases. we have four pieces of evidence proves that this mutation can lead to LCA-1: 1- Only this mutation was detected by WES and could be the main cause of LCA-1. 2-Bioinformatics tools such as SIFT, PolyPhen-2 and Mutation taster confirmed that this variant was predicted to be damaging and disease-causing. 3-According to the samples were analyzed by direct Sanger sequencing (Fig. 1B), the presence of this mutation was proved in the patient, and the pattern of inheritance must be an autosomal recessive for the GUCY2D gene because the patient carries a homozygous mutation and her consanguine parents are heterozygous for the detected mutation. 4- $A$ point mutation c.2348T>C of the GUCY2D gene, in exon 12 causes substitution of leucine by proline at position 783 which would be expected to affect retGC-1 function.

\section{Conclusion}

The present study detected a case of LCA-1 with a novel homozygous GUCY2D gene mutation (c.2348T>C; p.L783P) in an Iranian girl from a heterozygous and carrier parents using WES technique. It can be concluded that this point of detected mutation is a novel mutational hotspot point that carried in 
patient ancestors. Moreover, our findings confirm previous reports that mutation in GUCY2D gene is associated with LCA disease and we show that this method can be useful for identifying rare causative genetic variants in LCA patients.

\section{Abbreviations}

ACMG: American College of Medical Genetics and Genomics; cGMP: Cyclic Guanosine Monophosphate; dbSNP: Single Nucleotide Polymorphism Database; ExAC: Exome Aggregation Consortium; gDNA: Genomic DNA; GTP: Guanosine Triphosphate; HGMD: Human Gene Mutation Database; LCA: Leber Congenital Amaurosis; NLP: No Light Perception; PCR: Polymerase Chain Reaction; PolyPhen-2: Polymorphism Phenotyping v2; RetGC-1: Retinal Guanylyl Cyclase-1; SIFT: Sort Intolerated From Tolerated; WES: Whole Exome Sequencing; VEPs: Visual Evoked Potentials.

\section{Declarations}

\section{Ethics approval and consent to participate}

Written informed consent was obtained from the parents of the patient, and the study was, according to the Ethics Committee of Iran's Ministry of Health and Medical Education guidelines; reference number is not available.

\section{Consent for publication}

Written informed consent was obtained from the parents of the patient for publication of this case report.

\section{Availability of data and materials}

The data that support the findings of this study are available from the corresponding author upon reasonable request.

\section{Competing interests}

The authors declared there is no conflict of interest.

\section{Funding}

There is no financial support.

\section{Authors' contributions}

MN made design of the study and wrote the manuscript. MN, AIA and JMA analyzed and interpreted the data. All authors have read and approved the final manuscript.

\section{Acknowledgments}


The authors would like to thank the family members for their participation in this study.

\section{Author details}

${ }^{1}$ Department of Genetics, Khuzestan Science and Research Branch, Islamic Azad University, Ahvaz, Iran.

${ }^{2}$ Department of Genetics, Ahvaz Branch, Islamic Azad University, Ahvaz, Iran. ${ }^{3}$ Department of Biology, College of Science, University of Basrah, Iraq. ${ }^{4}$ Department of Medical Genetics, School of Medicine, Ahvaz Jundishapur University of Medical Sciences, Ahvaz, Iran.

\section{References}

1. den Hollander Al, Roepman R, Koenekoop RK, Cremers FP. Leber congenital amaurosis: genes, proteins and disease mechanisms. Prog Retin Eye Res. 2008;27(4):391-419. https://doi.org/10.1016/j.preteyeres.2008.05.003

2. Koenekoop RK. An overview of Leber congenital amaurosis: a model to understand human retinal development. Surv Ophthalmol. 2004;49(4):379-98. https://doi.org/10.1016/j.survophthal.2004.04.003

3. Imani S, Cheng J, Mobasher-Jannat A, Wei C, Fu S, Yang L et al. Identification of a novel RPGRIP1 mutation in an Iranian family with leber congenital amaurosis by exome sequencing. $\mathrm{J}$ Cell Mol Med. 2018;22(3):1733-1742. https://doi.org/10.1111/jcmm.13454

4. Foster A, Gilbert C. Epidemiology of childhood blindness. Eye (Lond). 1992;6(2):173-6. https://doi.org/10.1038/eye.1992.34

5. Rim PH, Magna LA, Ramalho AS. Genetics and prevention of blindness. Arq Bras Oftalmol. 2006;69(4):481-5. https://doi.org/10.1590/s0004-27492006000400005

6. Tabbara KF, Badr IA. Changing pattern of childhood blindness in Saudi Arabia. Br J Ophthalmol. 1985;69(4):312-5. https://doi.org/10.1136/bjo.69.4.312

7. Hozan I. Ibrahim, Hazha J. Hidayat. Case Report: A GUCY2D Gene Mutation in Kurdish Nation With Leber Congenital Amaurosis-1 Disease. Annals of RSCB. 2021;25(4):14049-54.

8. Salehi Chaleshtori AR, Garshasbi M, Salehi A. A novel deletion mutation in GUCY2D gene may be responsible for Leber congenital amaurosis-1 disease: A case report. J Curr Ophthalmol. 2019;31(4):458-462. https://doi.org/10.1016/j.joco.2019.07.002

9. Wimberg H, Lev D, Yosovich K, Namburi P, Banin E, Sharon D et al. Photoreceptor Guanylate Cyclase (GUCY2D) Mutations Cause Retinal Dystrophies by Severe Malfunction of Ca2+-Dependent Cyclic GMP Synthesis. Front Mol Neurosci. 2018;11:348. https://doi.org/10.3389/fnmol.2018.00348

10. Garcia-Hoyos M, Auz-Alexandre CL, Almoguera B, Cantalapiedra D, Riveiro-Alvarez R, Lopez-Martinez MA et al. Mutation analysis at codon 838 of the Guanylate Cyclase 2D gene in Spanish families with autosomal dominant cone, cone-rod, and macular dystrophies. Mol Vis. 2011;17:1103-9.

11. Yang Y, Muzny DM, Xia F, Niu Z, Person R, Ding Y et al. Molecular findings among patients referred for clinical whole-exome sequencing. JAMA. 2014;312(18):1870-9. 
http://doi.org/10.1001/jama.2014.14601

12. Chacon-Camacho OF, Zenteno JC. Review and update on the molecular basis of Leber congenital amaurosis. World J Clin Cases. 2015;3(2):112-24. https://doi.org/10.12998/wjcc.v3.i2.112

13. Feng $X$, Wei T, Sun J, Luo Y, Huo Y, Yu P et al. The pathogenicity of novel GUCY2D mutations in Leber congenital amaurosis 1 assessed by HPLC-MS/MS. PLoS One. 2020;15(4):e0231115. https://doi.org/10.1371/journal.pone.0231115

14. Gradstein L, Zolotushko J, Sergeev YV, Lavy I, Narkis G, Perez Y et al. Novel GUCY2D mutation causes phenotypic variability of Leber congenital amaurosis in a large kindred. BMC Med Genet. 2016;17:52. https://doi.org/10.1186/s12881-016-0314-2

\section{Figures}

(A)

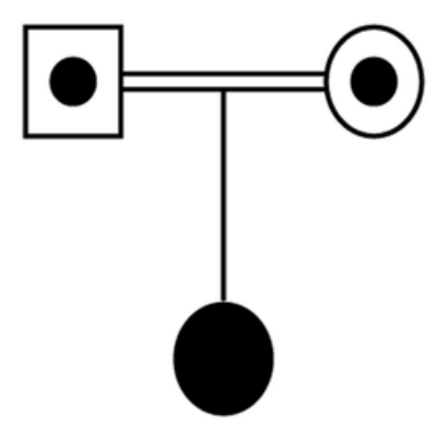

(C)

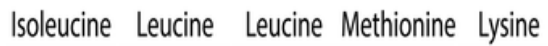

(B)

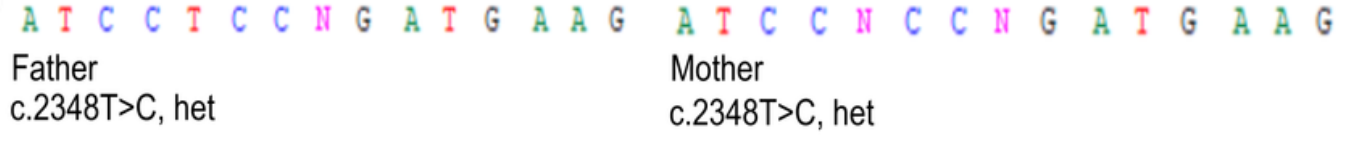

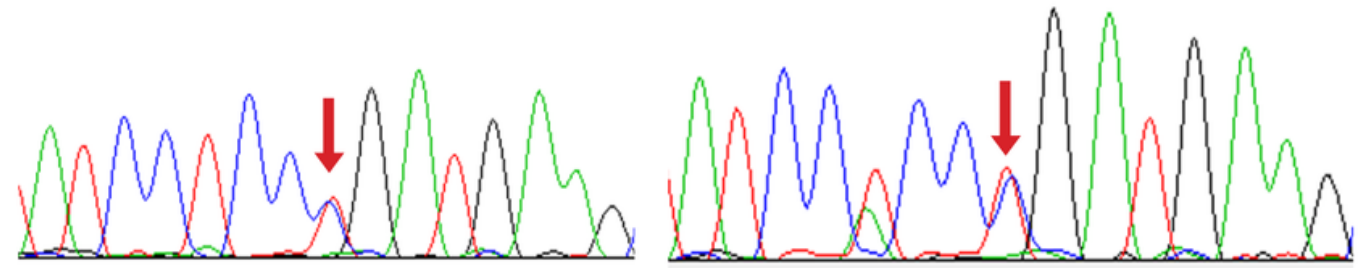

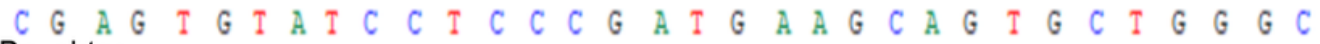
Daughter c. $2348 \mathrm{~T}>\mathrm{C}$, hom

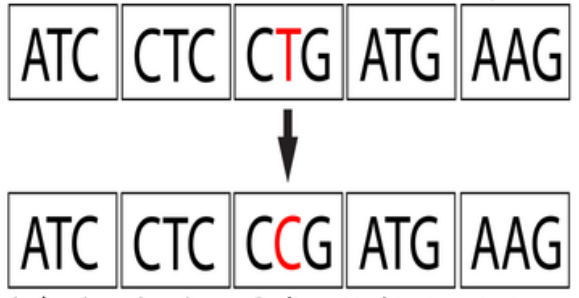

Isoleucine Leucine Proline Methionine Lysine

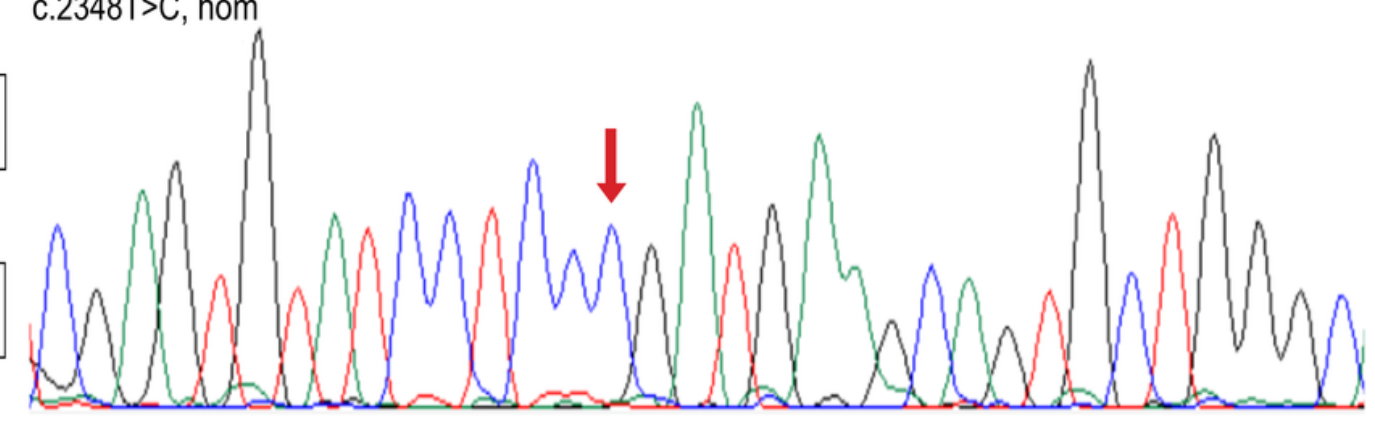

\section{Figure 1}

(A) Pedigree of the family with the p.L783P mutation. Symbols with a black dot at the center are carriers of a mutation but with no LCA-1 phenotype and filled symbol indicate an affected individual. (B) Electropherogram of the affected patient and her normal parents demonstrating a homozygous c.2348T $>$ C mutation of the GUCY2D gene and heterozygous carrier state in her parents. (C) The amino acid changes (leucine to proline) caused by the changes in the DNA sequence. 